\title{
Can XLIF Surgery Treat the Lumbar Spine Infection?
}

\section{Qingyi He*}

Orthopedic Department, Southwest Hospital, Third Military Medical University, China

\section{Description}

Hematogenous vertebral osteomyelitis has increased in recent years, likely due to longer life expectancies, higher prevalence of chronic disease, better diagnostic techniques, and more frequent use of indwelling intravascular catheters and immunosuppressive therapy [1]. Vertebral osteomyelitis represents $2 \%$ to $7 \%$ of all cases of osteomyelitis and approximately $50 \%$ with spine infections are older than 50 years, and two thirds are male [2]. It is associated with significant morbidity, including prolonged antimicrobial therapy, risk of recurrence, and decreased functional status. Staphylococcus aureus is among the most common organisms causing hematogenous vertebral osteomyelitis [1]. Medical management is the first choice to spondylodiscitis, patients who fail antibiotic therapy and bracing, or present with instability, significant deformity, neurologic deficits, or sepsis, were required operative debridement and stabilization [2]. ALIF (anterior lumbar interbody fusion), PLIF (posterior lumbar interbody fusion) and TLIF (transforaminal lumbar interbody fusion) were performed to treat lumbar spondylodiscitis respectively to achieve rather good clinical results but also causing much morbid [3-7].

XLIF (extreme lateral interbody fusion) is a minimally invasive, lateral, transpsoas approach for lumbar interbody fusion and is an alternative approach to ALIF [8]. Generally speaking, XLIF was used to treat degenerative disease, deformity, trauma, tumor, and total disk replacement has also been achieved via this technique [9]. Recently, XLIF was a minimally invasive approach surgery, sparing the anterior longitudinal ligament, and allowing sufficient visualization of the intervertebral discs and bodies to debride and place a large, lordotic cage in thoracic or lumbar spine infection [10,11]. Blizzard [12] et al. treated 11 spondylodiscitis patients with XLIF for average follow-up 9.3 months. All patients had improvements in pain and neurological symptoms. Fusion was confirmed with CT scans in five of six patients. At the last follow-up, all patients had normalization of inflammatory markers, no symptoms of infection was occurred, and none required repeat surgical treatment for spondylodiscitis. In our experience treated 12 cases of pyogenic lumbar vertebral osteomyelitis with XLIF, the minimal invasive spine surgery bears the several advantages including less tissue dissection, smaller incisions, decreased operative time (58 \pm $17 \mathrm{~min})$, estimated blood loss $(95 \pm 32 \mathrm{ml})$, shorter hospital stay (15 \pm $5 \mathrm{~d}$ ), reduced postoperative pain, which showed a promising future in surgery options in vertebral osteomyelitis.

Apart from osteomyelitis, XLIF may play some possible role in vertebral abscess, brucellar spondylodiscitis and lumbar spine tuberculosis, but there is little evidence to support the assumption. Because of the very few references in treatment of lumbar spine infection with XLIF, until now, there is no report of persistence or recurrence infection rates after XLIF including our cases. The indications of XLIF in treatment lumbar spine infection was one or two levels osteomyelitis, which can be done through one small incision. The contraindications to select the XLIF procedure in order to treat lumbar spine infection were severe destroyed vertebral body ( $>40 \%$ vertebral body), aggravated deformity $\left(>30^{\circ}\right.$ cobb angle in sagittal or coronal plane), multi- levels ( $\geqq 3$ levels) of osteomyelitis and mass of pus. Some special complications happened in XLIF. Eight to $10 \%$ of patients experience some initial psoas weakness which typically resolves within 1-2 weeks. The prevalence of sensory deficits was $1.6 \%$, psoas mechanical deficit was $1.6 \%$ and lumbar plexus related deficits was $2.9 \%$, these symptoms were generally resolved in about 6 weeks [13]. Proper patient selection, thorough knowledge of the anatomy, meticulous surgical technique and use of intraoperative monitoring can help avoid or decrease these complications [14].

\section{Acknowledgments}

This work was supported by grants from the Chongqing Application and Development Fund (cstc2013yykfA10008), China's Ministry of Health Fund (W2013ZT150), Southwest Hospital Clinical Innovation Fund (SWH2013LC25, WSS-2012-05)

\section{References}

1. Park KH, Cho OH, Jung M, Suk KS, Lee JH, et al. (2014) Clinical characteristics and outcomes of hematogenous vertebral osteo-myelitis caused by gramnegativebacteria. J Infect 69: 42-50.

2. Sapico FL, Montgomerie JZ (1979) Pyogenic vertebral osteomyelitis: report of nine cases and review of the literature. Clin Infect Dis 1: 754-776.

3. Dai LY, Chen WH, Jiang LS (2008) Anterior instrumentation for the treatment of pyogenic vertebral osteomyelitis of thoracic and lumbar spine. Eur Spine J 17: 1027-1034.

4. Ballard JL, Carlson G, Chen J, White J (2014) Anterior thoracolumbar spine exposure: critical review and analysis. Ann Vasc Surg 28: 465-469.

5. Fantini GA, Pappou IP, Girardi FP, Sandhu HS, Cammisa FP Jr (2007) Major vascular injury during anterior lumbar spinal surgery: incidence, risk factors, and management. Spine 32: 2751-2758.

6. Lee JS, Suh KT (2006) Posterior lumbar interbody fusion with an autogenous iliac crest bone graft in the treatment of pyogenic spondylodiscitis. J Bone Joint Surg $\mathrm{Br}$ 88: 765-770.

7. Lu ML, Niu CC, Tsai TT, Fu TS, Chen LH, et al. (2015) Transforaminal lumbar interbody debridement and fusion for the treatment of infective spondylodiscitis in the lumbar spine. Eur Spine J 24: 555-560.

8. Berjano P, Lamartina C (2011) Minimally invasive lateral transpsoas approach with advanced neurophysiologic monitoring for lumbar interbody fusion. Eur Spine J 20: 1584-1586

9. He Q (2014) The Extreme Lateral Interbody Fusion (XLIF): Its Today and Tomorrow. J Spine 3: e112.

10. Arnold PM, Anderson KK, McGuire RA Jr (2012) The lateral transpsoas approach to the lumbar and thoracic spine: A review. Surg Neurol Int 3: S198-S215.

11. Karikari IO, Nimjee SM, Hardin CA, Hughes BD, Hodges TR, et al. (2011) Extreme lateral interbody fusion approach for isolated thoracic and

*Corresponding author: Qingyi He, Orthopedic Department, Southwest Hospital, Third Military Medical University, 30 Gao Tan Yan Street, Sha Ping Ba District, Chongqing, 400038, China, Tel: +8602365340297; E-mail: qingyihe.hq@gmail.com

Received September 02, 2015; Accepted September 23, 2015; Published September 30, 2015

Citation: Qingyi H (2015) Can XLIF Surgery Treat the Lumbar Spine Infection? J Trauma Treat 4: 264. doi:10.4172/2167-1222.1000264

Copyright: (C) 2015 Qingyi H. This is an open-access article distributed under the terms of the Creative Commons Attribution License, which permits unrestricted use, distribution, and reproduction in any medium, provided the original author and source are credited. 
thoracolumbar spine diseases: initial clinical experience and early outcomes. J Spinal Disord Tech 24: 368-375.

12. Blizzard DJ, Hills CP, Isaacs RE, Brown CR (2015) Extreme lateral interbody fusion with posterior instrumentation for spondylodiscitis. J Clin Neurosci In Press.

13. Pumberger M, Hughes AP, Huang RR, Sama AA, Cammisa FP, et al. (2012)
Neurologic deficit following lateral lumbar interbody fusion. Eur Spine J 21 1192-1199.

14. Hood B, Vanni S (2010) Minimally Invasive Extreme Lateral Trans-Psoas Approach to the Lumbar Spine: Applications and Techniques, Spine Surgery, University of Miami, Miller School of Medicine, Jackson Memorial Hospital, Miami, FL, USA. 